\title{
Reconstruction of a 2D seismic wavefield by seismic gradiometry
}

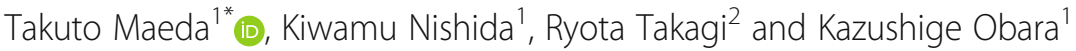

\begin{abstract}
We reconstructed a 2D seismic wavefield and obtained its propagation properties by using the seismic gradiometry method together with dense observations of the Hi-net seismograph network in Japan. The seismic gradiometry method estimates the wave amplitude and its spatial derivative coefficients at any location from a discrete station record by using a Taylor series approximation. From the spatial derivatives in horizontal directions, the properties of a propagating wave packet, including the arrival direction, slowness, geometrical spreading, and radiation pattern can be obtained. In addition, by using spatial derivatives together with free-surface boundary conditions, the 2D vector elastic wavefield can be decomposed into divergence and rotation components. First, as a feasibility test, we performed an analysis with a synthetic seismogram dataset computed by a numerical simulation for a realistic 3D medium and the actual Hi-net station layout. We confirmed that the wave amplitude and its spatial derivatives were very well-reproduced for period bands longer than $25 \mathrm{~s}$. Applications to a real large earthquake showed that the amplitude and phase of the wavefield were well reconstructed, along with slowness vector. The slowness of the reconstructed wavefield showed a clear contrast between body and surface waves and regional non-great-circle-path wave propagation, possibly owing to scattering. Slowness vectors together with divergence and rotation decomposition are expected to be useful for determining constituents of observed wavefields in inhomogeneous media.
\end{abstract}

Keywords: Seismic gradiometry, Array signal processing, Surface waves, Scattering, Numerical simulation

\section{Introduction}

The High-sensitivity seismograph network (Hi-net), which is operated by the National Research Institute for Earth Science and Disaster Resilience of Japan (Okada et al. 2004), is one of the densest seismograph networks in the world. It covers almost all of the Japanese islands with a nearly uniform station separation of about $20 \mathrm{~km}$ (Fig. 1). Although the Hi-net stations are equipped with short-period seismometers with a natural frequency of $1 \mathrm{~Hz}$ at the bottom of boreholes, the high sensitivity, low noise level, and wide dynamic range of the recording systems allow us to reconstruct long-period broadband records. Our previous experiments suggest that seismic waves with periods of up to $100 \mathrm{~s}$ generated by large far-field earthquakes can be used for data analysis with appropriate correction for sensor sensitivity (Maeda et al. 2011).

\footnotetext{
* Correspondence: maeda@eri.u-tokyo.ac.jp

${ }^{1}$ Earthquake Research Institute, The University of Tokyo, 1-1-1 Yayoi,

Bunkyo-ku, Tokyo 113-0032, Japan

Full list of author information is available at the end of the article
}

Observations of long-period seismic waves by dense arrays capture seismic ground motion directly as spatially propagating waves, rather than as individual traces at discrete stations. Thus, seismic waves propagating across arrays have been visualized for various arrays (e.g., Obara et al. 2005; Sheldrake et al. 2002; Trabant et al. 2012). Such visualization is useful not only for educational or outreach purposes but also for capturing characteristic wave propagation. By treating a seismic wave as a spatially varying field, Maeda et al. (2014) detected a new sort of long-period scattered wave that originated from reverberation in the seawater column within a deep trench.

In this study, we applied a relatively new technique for treating a large set of array data, called seismic gradiometry, to reconstruct and characterize a seismic wavefield observed by the Hi-net array. The seismic gradiometry technique (Langston 2007a) was originally developed as a method to measure the spatial gradient of seismic waves observed by dense arrays. Langston (2007a) showed that the slowness could be directly estimated from the spatial 


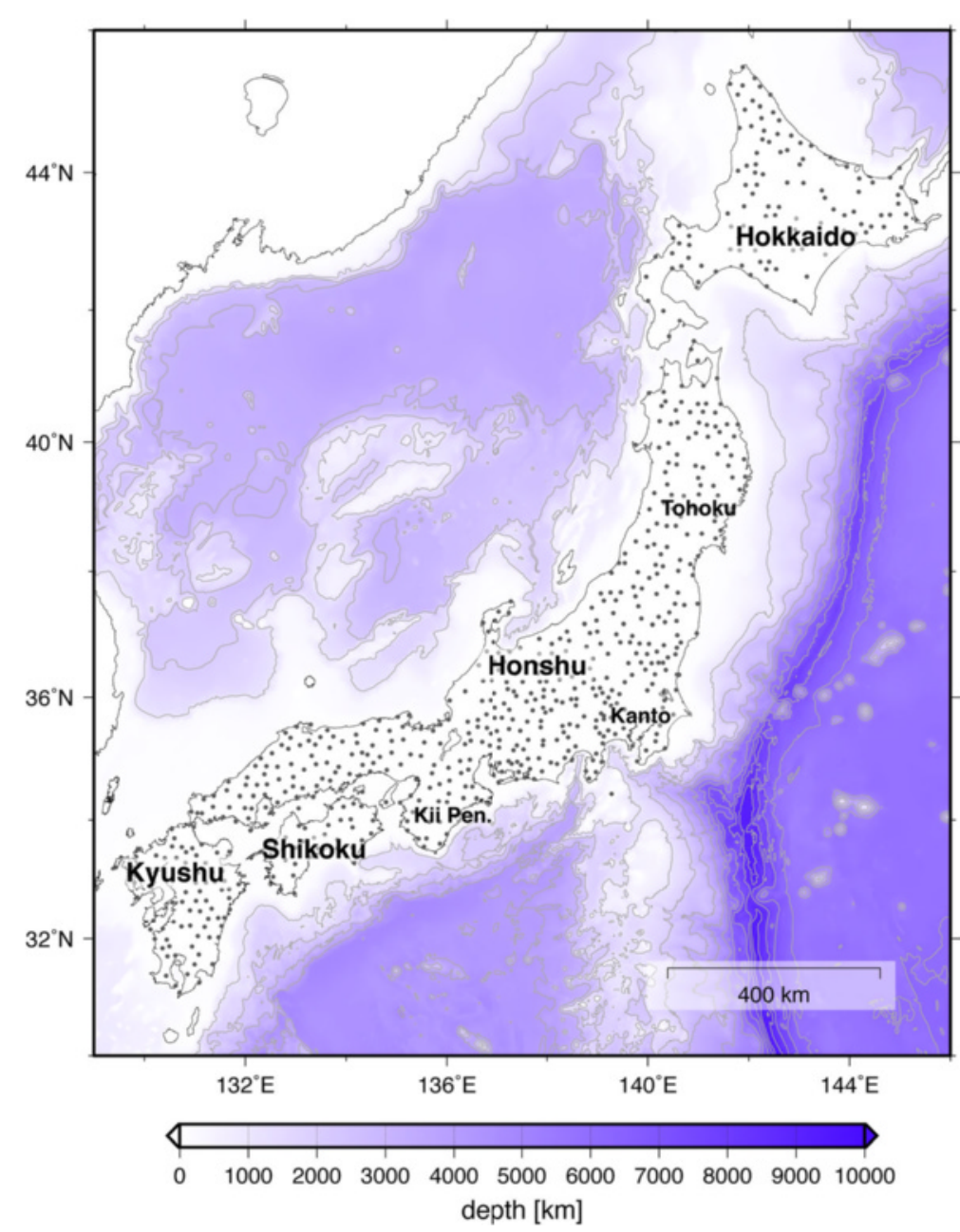

Fig. 1 Index map showing the Hi-net station distribution (dots) used in this study and the names of major Japanese islands and districts. Contours show bathymetric depth at $1000 \mathrm{~m}$ intervals

gradient. Igel et al. (2005) proposed a similar approach for the analysis of records observed by rotational seismometers. Subsequently, the seismic gradiometry technique was further developed for application to a two-dimensional wave propagation problem (Langston 2007b) and to polarized waves (Langston and Liang 2008) and to improve its stability in the time domain (Langston 2007c). Compared with traditional array methods such as the semblance method (Neidell and Taner 1971), seismic gradiometry has the advantage that it estimates slowness as a spatially varying value, whereas other array methods usually assume a homogeneous plane wave incidence. In contrast to traditional array methods, which utilize phase differences for slowness estimation, seismic gradiometry models both the amplitude and phase of the observed wavefield. The possible separation of non-plane wave characteristics such as the radiation pattern and geometrical spreading is also a unique feature of the method.
In this paper, we first show that seismic gradiometry is a useful tool for reconstructing a spatially continuous seismic wavefield, although the method was originally applied (Liang and Langston 2009) to the estimation of the phase speed of surface waves at individual stations. We also propose that the divergence and rotation of the three-component seismic wavefield can be estimated by seismic gradiometry and used to characterize seismic wave propagation features. After synthetic tests using three-dimensional numerical simulation results for an inhomogeneous structure model beneath Japan, we apply the proposed method to seismic waves in longperiod bands ( $>25 \mathrm{~s}$ ) observed by Hi-net stations.

\section{Methods/Experimental}

Seismic gradiometry uses a Taylor series expansion of the seismic wavefield in two-dimensional (2D) horizontal space. If we have observed waveform $u^{\text {obs }}$ at location 
$\left(x_{S}, y_{S}\right)$ and time $t$, the wave $u$ at a nearby location $\left(x_{G}, y_{G}\right)$ can be approximated by a first-order approximation of the Taylor series:

$$
\begin{aligned}
& u^{\mathrm{obs}}\left(x_{S}, y_{S} ; t\right) \cong u\left(x_{G}, y_{G} ; t\right) \\
& +\frac{\partial u\left(x_{G}, y_{G} ; t\right)}{\partial x}\left(x_{S}-x_{G}\right) \\
& +\frac{\partial u\left(x_{G}, y_{G} ; t\right)}{\partial y}\left(y_{S}-y_{G}\right) .
\end{aligned}
$$

By using several observations from stations near the target grid at locations $\left(x_{S i}, y_{S i}\right)(i=1, \cdots, N)$, we can construct an observation equation of the wave amplitude for a given location as follows:

$$
\begin{aligned}
& \boldsymbol{u}^{\mathrm{obs}}=\boldsymbol{G m} \\
& \boldsymbol{u}^{\mathrm{obs}} \equiv\left(\begin{array}{c}
u^{\mathrm{obs}}\left(x_{S 1}, y_{S 1} ; t\right) \\
u^{\mathrm{obs}}\left(x_{S 2}, y_{S 2} ; t\right) \\
\vdots \\
u^{\mathrm{obs}}\left(x_{S N}, y_{S N} ; t\right)
\end{array}\right) \\
& \boldsymbol{G} \equiv\left(\begin{array}{ccc}
1 & x_{S 1}-x_{G} & y_{S 1}-y_{G} \\
1 & x_{S 2}-x_{G} & y_{S 2}-y_{G} \\
\vdots \\
1 & x_{S N}-x_{G} & y_{S N}-y_{G}
\end{array}\right) \\
& \boldsymbol{m} \equiv\left(\begin{array}{c}
u\left(x_{G}, y_{G} ; t\right) \\
\partial_{x} u\left(x_{G}, y_{G} ; t\right) \\
\partial_{y} u\left(x_{G}, y_{G} ; t\right)
\end{array}\right),
\end{aligned}
$$

where $N$ is the number of stations used for the estimation. Liang and Langston (2009) used a very similar observation equation to estimate Rayleigh waves for the case that the grid locations are collocated with seismic stations. Though their approach simplifies gradiometry Eq. (2), the estimation of the seismic wavefield is limited to the station location. In our approach, we use seismic gradiometry to reconstruct the seismic wavefield as a continuous 2D field irrespective of the station location. We note that Spudich et al. (1995) performed a similar series expansion to estimate surface strain and stress tensors for the 1992 Landers earthquake (M7.4) recorded by a dense array.

The wave amplitude and its spatial gradients can be estimated by using the least squares method if we have input data from three or more stations. We note that we can significantly reduce the computational cost by using a previously computed kernel matrix for the least squares calculation because this inverse problem depends only on the station layout:

$$
\boldsymbol{m}=\left(\boldsymbol{G}^{T} \boldsymbol{W} \boldsymbol{G}\right)^{-1} \boldsymbol{G}^{T} \boldsymbol{W} \boldsymbol{u}^{\mathrm{obs}} \equiv \boldsymbol{K} \boldsymbol{u}^{o b s},
$$

where $W$ is a diagonal weight matrix (e.g., Menke 2012). As a weighting factor, we empirically adopted the Gaussian function, which smoothly decreases weight amplitude with distance between the target grid point and the station with a variance of $\sigma^{2}=\Delta_{0}^{2} / 10$, where $\Delta_{0}=50 \mathrm{~km}$ is a cutoff distance. Equation (3) can be formally decomposed as follows:

$$
\begin{gathered}
u\left(x_{G}, y_{G} ; t\right)=\boldsymbol{k}_{\mathrm{u}} \cdot \boldsymbol{u}^{\mathrm{obs}} \\
\partial_{x} u\left(x_{G}, y_{G} ; t\right)=\boldsymbol{k}_{\partial x} \cdot \boldsymbol{u}^{\mathrm{obs}} \\
\partial_{y} u\left(x_{G}, y_{G} ; t\right)=\boldsymbol{k}_{\partial y} \cdot \boldsymbol{u}^{\mathrm{obs}}
\end{gathered}
$$

where $\boldsymbol{K} \equiv\left[\boldsymbol{k}_{u}, \boldsymbol{k}_{\partial x}, \boldsymbol{k}_{\partial y}\right]^{T}$ is a part of the solution of Eq. (3). Equation (4) suggests that the displacement and its spatial gradients at a grid point are obtained from weighted averages of the observed waveform at nearby stations, where the weighting factor $K$ is obtained from the station layout. We note that this is an over-determined problem when three or more stations are used. Therefore, regularization by smoothing or damping is not necessary to solve this problem.

The quality of the estimation of the displacement and its spatial derivatives strongly depends on the locations of the stations relative to the grid point. The quality of the estimation improves as the number of stations used for Eq. (3) increases. On the other hand, the use of more distant stations may result in underestimation of the spatial derivatives of the waves, because we assumed a first-order Taylor series expansion of the wavefield in Eq. (1) (Langston 2007a). We therefore used only those stations within $50 \mathrm{~km}$, with a smoothly decreasing weighting factor.

\section{Love and Rayleigh wave decomposition}

By using a three-component seismogram and its derivatives, we can estimate the divergence and rotation of elastic vector motion. To eliminate the derivative with respect to depth, we used a traction-free boundary condition as proposed by Shapiro et al. (2000),

$$
\begin{aligned}
& \operatorname{div} \boldsymbol{u}=\frac{\partial u_{x}}{\partial x}+\frac{\partial u_{y}}{\partial y}+\frac{\partial u_{z}}{\partial z}=\frac{2 \mu}{\lambda+2 \mu}\left(\frac{\partial u_{x}}{\partial x}+\frac{\partial u_{y}}{\partial y}\right) \simeq \frac{2}{3}\left(\frac{\partial u_{x}}{\partial x}+\frac{\partial u_{y}}{\partial y}\right) \\
& \operatorname{rot} \boldsymbol{u}=\left[\frac{\partial u_{z}}{\partial y}-\frac{\partial u_{y}}{\partial z}, \frac{\partial u_{x}}{\partial z}-\frac{\partial u_{z}}{\partial x}, \frac{\partial u_{y}}{\partial x}-\frac{\partial u_{x}}{\partial y}\right]=\left[2 \frac{\partial u_{z}}{\partial y},-2 \frac{\partial u_{z}}{\partial x}, \frac{\partial u_{y}}{\partial x}-\frac{\partial u_{x}}{\partial y}\right]
\end{aligned}
$$

where $\lambda$ and $\mu$ are Lamé coefficients. We approximated the estimation of divergence by assuming a Poisson solid $(\lambda=\mu)$. The displacement vector can be represented by using potentials $\phi, X$, and $\psi$ as 


$$
\boldsymbol{u}=\operatorname{grad} \phi+\operatorname{rot}\left(\begin{array}{l}
0 \\
0 \\
\chi
\end{array}\right)+\operatorname{rot} \operatorname{rot}\left(\begin{array}{l}
0 \\
0 \\
\psi
\end{array}\right)
$$

where the terms on the right-hand side correspond to $P_{-}$, $S H$-, and $S V$-motion, respectively (Aki and Richards 2002). The divergence and rotation components are therefore written as

$$
\begin{aligned}
\operatorname{div} \boldsymbol{u} & =\nabla^{2} \phi \\
\operatorname{rot} \boldsymbol{u} & =\left(\begin{array}{c}
\partial_{x} \partial_{z} \chi-\partial_{y}\left(\nabla^{2} \psi\right) \\
\partial_{y} \partial_{z} \chi+\partial_{x}\left(\nabla^{2} \psi\right) \\
-\left(\partial_{x}^{2}+\partial_{y}^{2}\right) \chi
\end{array}\right) .
\end{aligned}
$$

The divergence and the vertical component of the rotation vector therefore consist of Rayleigh $(P-S V)$ and Love $(S H)$ waves, respectively, whereas the horizontal components of the rotation are a mixture of Love and Rayleigh waves. By estimating these terms by seismic gradiometry, we can separate the Love and Rayleigh wave components from the observed ground velocity or displacement signal.

\section{Slowness estimation}

By modeling the observed displacement as the amplitude term multiplied by the propagation phase shift term, we can separate a term related to the geometrical spreading and radiation pattern from the slowness vector. To estimate the arrival direction, we need to know ground velocity, displacement, and its spatial derivatives. Following Langston (2007b), we model the observed displacement as follows:

$$
u(x, y ; t)=G(x, y) f\left[t-p_{x}\left(x-x_{0}\right)-p_{y}\left(y-y_{0}\right)\right]
$$

where $G$ denotes the amplitude term and $x_{0}$ and $y_{0}$ are a reference location. By taking derivatives with respect to spatial variables, we obtain

$$
\begin{aligned}
& \frac{\partial u(x, y ; t)}{\partial x}=A_{x}(x) u(x, y ; t)+B_{x}(x) v(x, y ; t) \\
& \frac{\partial u(x, y ; t)}{\partial y}=A_{y}(x) u(x, y ; t)+B_{y}(x) v(x, y ; t),
\end{aligned}
$$

where $v(x, y ; t)=\partial_{t} u(x, y ; t)$ is ground velocity, obtained from the derivative of the ground displacement wavefield with respect to time, and coefficients $A$ and $B$ (hereinafter referred to as the gradiometry parameters) are defined as follows:

$$
\begin{aligned}
& A_{x}(x) \equiv \frac{1}{G(x, y)} \frac{\partial G(x, y)}{\partial x}, A_{y}(y) \equiv \frac{1}{G(x, y)} \frac{\partial G(x, y)}{\partial y} \\
& B_{x}(x) \equiv-\left[p_{x}+\frac{\partial p_{x}}{\partial x}\left(x-x_{0}\right)\right] \simeq-p_{x}, B_{y}(y) \equiv-\left[p_{y}+\frac{\partial p_{y}}{\partial y}\left(y-y_{0}\right)\right] \simeq-p_{y} .
\end{aligned}
$$

Notice that the spatial derivatives of the slowness vector are omitted in Eq. (10). In other words, we postulated that the slowness field changes slowly and that it can be assumed that the slowness is nearly constant at a grid point and the surrounding stations used for gradiometry estimation.

Several methods, for example Fourier domain (Langston 2007a) or analytic signal (Langston 2007c; Liang and Langston 2009) methods, have been proposed for estimating these coefficients. Here, we propose a simple alternative method for estimation of the gradiometry parameters. We use a time window consisting of $M$ discretized time samples, and assume that the parameters $A_{i}$ and $B_{i}(i=x, y)$ do not change within the time window:

$$
\begin{gathered}
\partial_{i} u\left(x, y ; t_{1}\right)=A_{i}(x) u\left(x, y ; t_{1}\right)+B_{i}(x) v\left(x, y ; t_{1}\right) \\
\partial_{i} u\left(x, y ; t_{2}\right)=A_{i}(x) u\left(x, y ; t_{2}\right)+B_{i}(x) v\left(x, y ; t_{2}\right) \\
\vdots \\
\partial_{i} u\left(x, y ; t_{M}\right)=A_{i}(x) u\left(x, y ; t_{M}\right)+B_{i}(x) v\left(x, y ; t_{M}\right) .
\end{gathered}
$$

From $M$ discrete time samples of data for ground displacement $u(x, y ; t)$, ground velocity $v(x, y ; t)$, and the estimated spatial gradient of ground displacement $\partial_{i} u(x, y ; t)$, the coefficients $A_{i}(x)$ and $B_{i}(x)$ are estimated by the least squares method. Because it is a simple two-parameter estimation, it has the following analytic solution:

$$
\begin{aligned}
A_{i}(x) & =\frac{\left(\boldsymbol{v}_{t} \cdot \boldsymbol{v}_{t}\right)\left(\partial_{i} \boldsymbol{u}_{t} \cdot \boldsymbol{u}_{t}\right)-\left(\boldsymbol{u}_{t} \cdot \boldsymbol{v}_{t}\right)\left(\partial_{i} \boldsymbol{u}_{t} \cdot \boldsymbol{v}_{t}\right)}{\left(\boldsymbol{u}_{t} \cdot \boldsymbol{u}_{t}\right)\left(\boldsymbol{v}_{t} \cdot \boldsymbol{v}_{t}\right)-\left(\boldsymbol{u}_{t} \cdot \boldsymbol{v}_{t}\right)^{2}} \\
B_{i}(x) & =\frac{\left(\boldsymbol{u}_{t} \cdot \boldsymbol{u}_{t}\right)\left(\partial_{i} \boldsymbol{u}_{t} \cdot \boldsymbol{v}_{t}\right)-\left(\boldsymbol{u}_{t} \cdot \boldsymbol{v}_{t}\right)\left(\partial_{i} \boldsymbol{u}_{t} \cdot \boldsymbol{u}_{t}\right)}{\left(\boldsymbol{u}_{t} \cdot \boldsymbol{u}_{t}\right)\left(\boldsymbol{v}_{t} \cdot \boldsymbol{v}_{t}\right)-\left(\boldsymbol{u}_{t} \cdot \boldsymbol{v}_{t}\right)^{2}}
\end{aligned}
$$

where $\boldsymbol{u}_{t}, \boldsymbol{v}_{t}$, and $\partial_{i} \boldsymbol{u}_{t}$ are vectors constituted by discrete time samples of ground displacement, ground velocity, and spatial gradients of ground displacement, respectively. We note that Liu and Holt (2015) used a very similar least squares method for estimating gradiometry parameters but with a damping constraint.

Because the estimation (12) is a pure time-domain method that uses a finite time window width, it does not require a long-duration time window as do methods using a Fourier transform (Langston 2007b) or an analytic signal with a Hilbert transform (Langston 2007c). The estimation should be stable except for the case in which the denominator of Eq. (12), $\left(\boldsymbol{u}_{t} \cdot \boldsymbol{u}_{t}\right)\left(\boldsymbol{v}_{t} \cdot \boldsymbol{v}_{t}\right)-\left(\boldsymbol{u}_{t} \cdot \boldsymbol{v}_{t}\right)^{2}$, is equal to or very close to zero. Such instability can occur 
only if the displacement time series $\boldsymbol{u}_{t}$ is parallel to the ground velocity $\boldsymbol{v}_{t}$. However, such a situation does not arise if a single wave packet arrives at the array as assumed in Eq. (8), because the ground velocity phase of a packet always differs from that of the displacement by $\pi / 2$. Thus, the gradiometry parameter estimation can be unstable only if multiple wave packets arrive at the array within the same time window. This drawback is intrinsic to the seismic gradiometry method itself, owing to the single wave packet assumption, not to the particular estimation method proposed here.

\section{Results}

\section{Feasibility test with numerical simulation}

For a feasibility test of wavefield estimation by seismic gradiometry, we first performed a synthetic test using the actual Hi-net station layout. A three-dimensional numerical simulation of seismic wave propagation was performed by using the finite difference method with the Japan Integrated Velocity Structure Model (JIVSM) of Koketsu et al. (2012) with a spatial extension to include an area not covered by the original model (Maeda et al. 2014). Technical details of the numerical simulation using the finite difference method are given by Maeda and Furumura (2013) and Maeda et al. (2013). We applied the seismic gradiometry method to synthetic seismograms at Hi-net stations to estimate the spatial derivatives of the seismic wavefield, and also to estimate the divergence and rotation of elastic motion. The spatial derivatives of seismic waves in every grid of the numerical model were obtained during the numerical simulation by the finite difference method. Therefore, we compared the spatial derivatives between the numerical simulation and that estimated from the seismic gradiometry method.

For the numerical simulation, we used a point source representation of the 2011 Tohoku Earthquake (Mw 9.0) by Kumagai et al. (2012) as example input. The sourcetime function was represented by single-cycle Küpper wavelets (Mavroeidis and Papageorgiou 2003) with a characteristic duration of $10 \mathrm{~s}$. The extended JIVSM model, which covered most of the Japanese archipelago, was discretized with a spatial grid spacing of $500 \mathrm{~m}$. Numerical integration of the equation of motion was performed with a time step of $0.025 \mathrm{~s}$. Because we used a relatively larger spatial grid size to cover a wider area and aimed to reproduce long-period waves with periods of $10-100 \mathrm{~s}$, we set the minimum wavespeed to $1.5 \mathrm{~km} / \mathrm{s}$; slower wavespeeds in the model were clipped. This model set-up is designed to reproduce seismic waves with periods longer than $5 \mathrm{~s}$. The simulation was performed in a coordinate system rotated horizontally $40^{\circ}$ in a clockwise direction so that it would effectively cover the whole Japanese archipelago while saving memory space (Fig. 2). From the numerical grids corresponding to the Hi-net station locations, ground velocity and ground displacement records were exported with a time step of $0.1 \mathrm{~s}$. Because we adopted an inhomogeneous JIVSM model rather than a simplified model, we could observe realistic, complicated wave behavior (Fig. 2, Additional file 1: Movie S1) that considerably affected the synthetic records.

The ground displacement and ground velocity traces were band-pass-filtered between 25 and $50 \mathrm{~s}$; then they were decimated to a sampling frequency of $1 \mathrm{~Hz}$. The grid points were prepared at $0.2^{\circ}$ intervals of the geographical coordinates. Because we observed that the gradiometry estimation outside of the station network was very poor, we limited the distribution of grid points to locations inside of Delaunay triangles having Hi-net station locations as triangle nodes. In addition, we required grid points to have three or more stations within the distance threshold $(50 \mathrm{~km})$ so that the estimation would be over-determined. The spatial extent of grid points with the number of used stations (Fig. 3) show that the grid points mostly covered the Japanese archipelago, but no grids were located very close to the shoreline, in particular, on the Japan Sea side where station coverage was sparse.

At every grid point, we estimated the spatial derivatives of the displacement by seismic gradiometry. We then calculated the divergence and rotation components of vector motion from these derivatives of the three-component seismograms. These divergence and rotation components were also calculated directly by finite difference simulation for comparison. In the estimation of divergence by seismic gradiometry, we used an approximation of Eq. (5) by assuming a Poisson solid $(\lambda=\mu)$ to mimic the real-world situation, in which the true inhomogeneous medium is unknown. In contrast, the exact divergence was calculated, including derivatives with respect to depth, in the case of the finite difference simulation in the same period band.

Time-lapse snapshots (Fig. 4) and a time-sequential movie (Additional file 2: Movie S2) show that the major characteristics of the divergence and rotation wavefields on land were well-reconstructed by the seismic gradiometry technique. Initially, the long-period $P$-wave appeared only in the divergence wavefield (mark A), whereas it was almost zero in the rotation wavefield (mark B). At a later elapsed time, the divergence and rotation wavefields contained coherent wave packets at different geographic locations (marks C and D) owing to different phase speeds; these wave packets correspond to Rayleigh and Love waves, respectively. Even long-period coda waves due to the heterogeneous medium structure, which appeared after passage of the Rayleigh waves, were reconstructed well (mark E), although the amplitude of the reconstructed wavefield is slightly weaker than the assumed amplitude. The amplitude difference in the coda wave part may be attributable to the shorter dominant wavelength. These results demonstrate that for this 


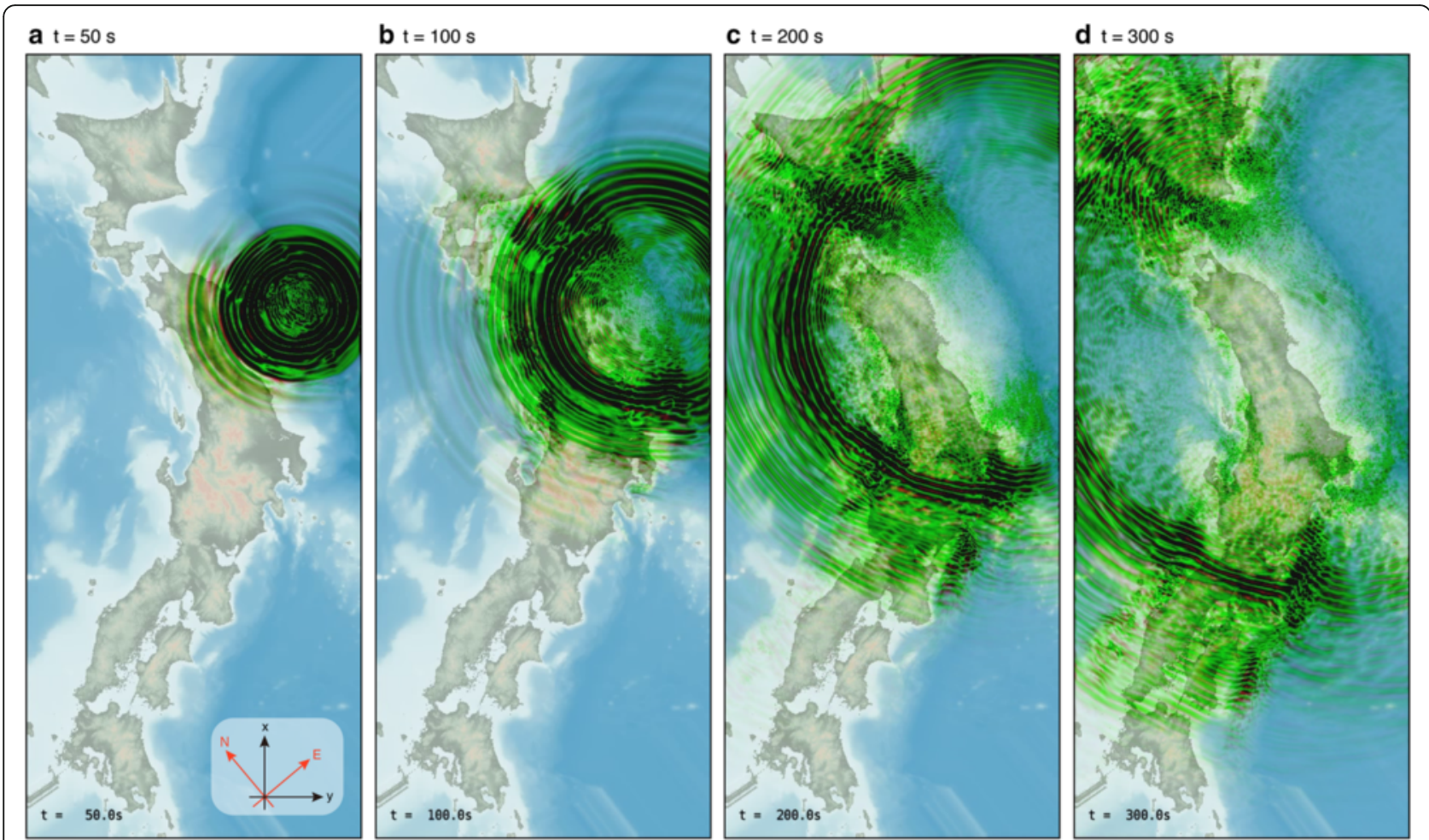

Fig. 2 Snapshots of the simulated seismic wavefield used as a reference solution of the numerical test of the seismic gradiometry (see text) at elapsed times of (a) $t=50$, (b) $t=100$, (c) $t=200 \mathrm{~s}$, and (d) $t=300 \mathrm{~s}$, measured from the start of the earthquake. Red and green colors overlaid on the topographic map show scaled divergence and the absolute value of the rotation vector of the velocity of ground motion at the ground surface or on the seafloor. The local Cartesian horizontal coordinates $(x, y)$ used in the simulation and the geographical directions $(N, E)$ are shown in a. An MP4-formatted movie of the time-dependent wavefield is included in Additional file 1: Movie S1)

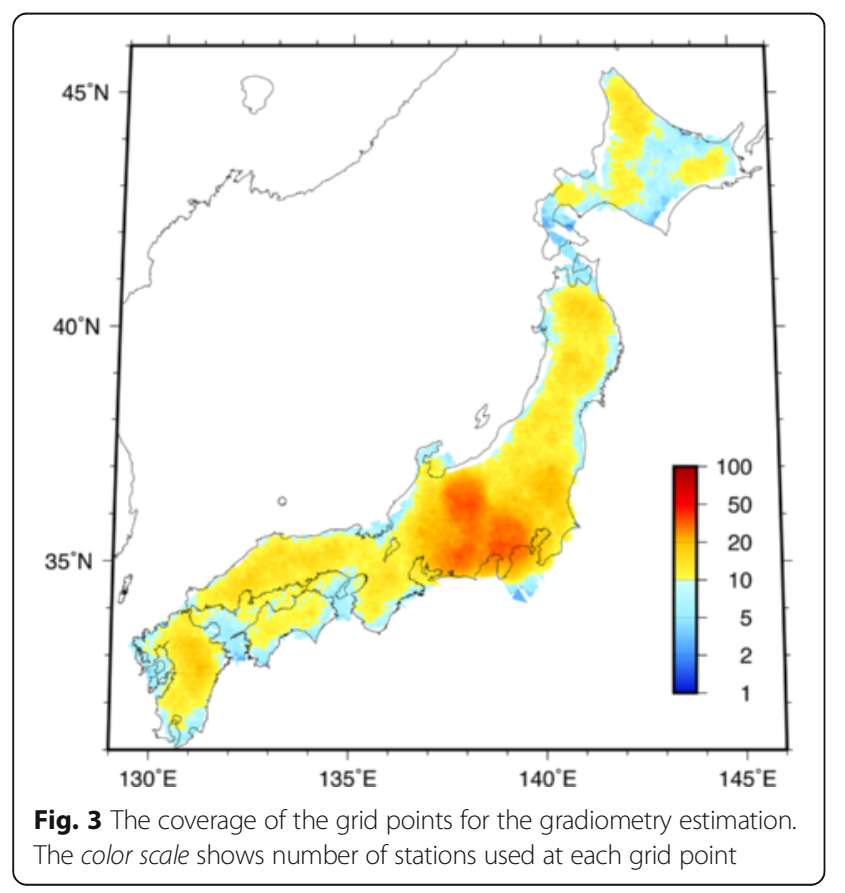

period range of 25-50 s, Rayleigh and Love waves (and body waves) with long periods can be decomposed from the three-component traces at Hi-net stations by seismic gradiometry.

A more quantitative comparison between the estimates and the reference solution was obtained by crosscorrelation of their divergence and rotation components in the time domain (Fig. 5). We used the whole time series up to $500 \mathrm{~s}$ of elapsed time after the earthquake to calculate the cross-correlations. In the target period band of $25-50 \mathrm{~s}$, the correlation coefficients were nearly 1.0, suggesting perfect recovery compared to the reference solution for periods longer than $25 \mathrm{~s}$, regardless of the number of stations (Fig. 3) used for the estimation. Trial applications using a shorter period band (10$20 \mathrm{~s}$; Fig. 6a) clearly failed to reproduce the divergence and rotation, but application to a longer period band of 50-100 s (Fig. 6b) also produced an almost perfect result. These results confirm that the ratio between the average station separation and the wavelength is a key parameter for reproducing a wavefield by seismic gradiometry. Two very minor modulations were seen around Hokkaido in the divergence component, and in Tohoku 


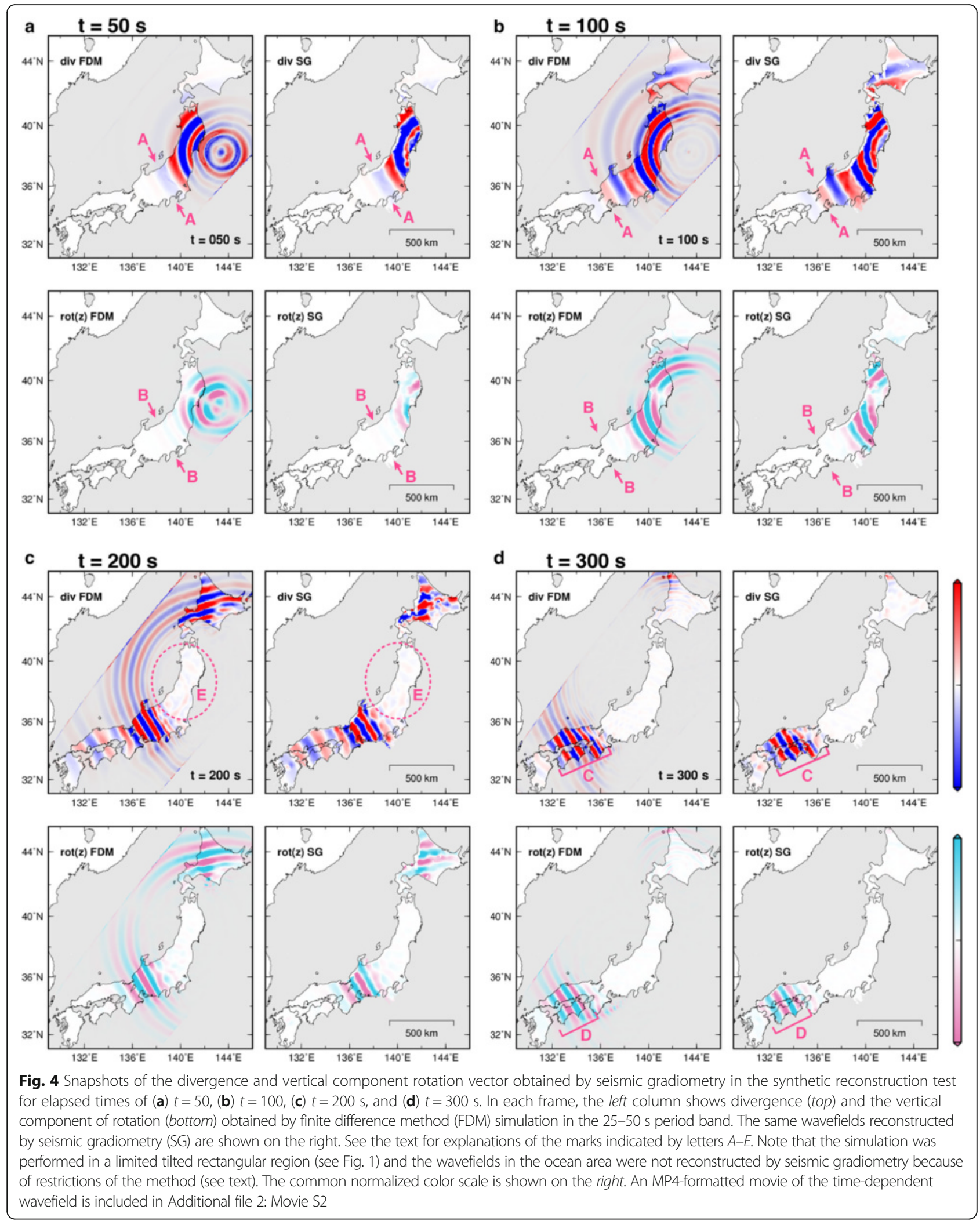



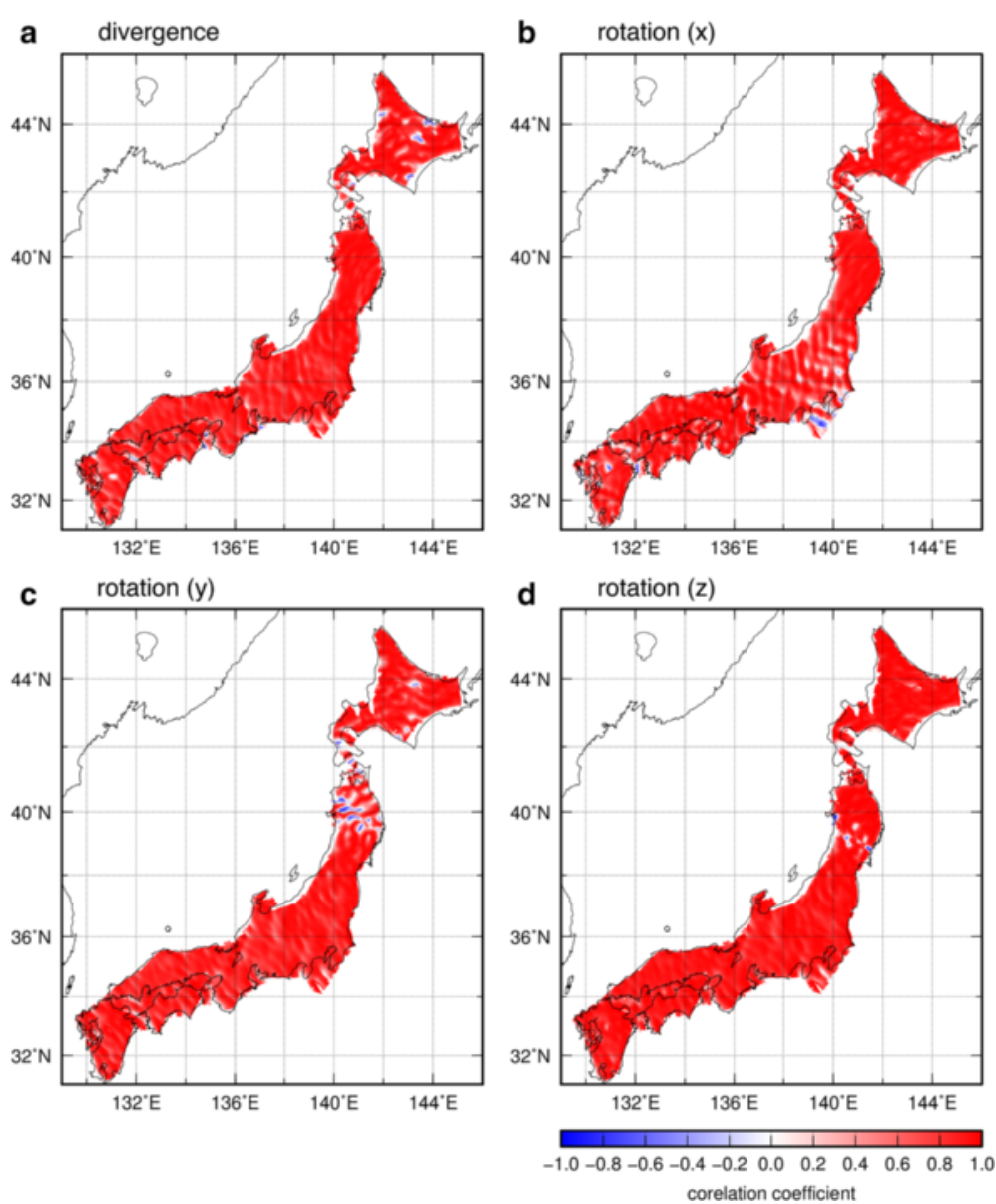

Fig. 5 Cross-correlation coefficients for divergence (a) and components of the rotation vectors (b-d) in grids between the wavefield obtained by numerical synthesis and that reconstructed by seismic gradiometry in the 25-50 s period band

district in the $y(\mathrm{SW}-\mathrm{NE})$ component, of the rotation (Fig. 4). The former may be due to the assumption of a Poisson solid to convert the derivative with respect to the vertical direction into that with respect to the horizontal. The latter may be attributable to the weak amplitude of Love waves in that region owing to the radiation pattern from the source (e.g., Stein and Wysession 2003, their Figure 4.3.12). The seismic gradiometry assumes a linear change of amplitude in nearby grids, but this assumption may not be valid around the nodal direction of the source radiation pattern, which may lead this modulation.

\section{Real-world application: 2005 Off-Tohoku outer-rise earthquake}

Next, we applied the same technique to real observations made during an earthquake that occurred in the east off Tohoku on 16 August 2005 (Mw 7.2). This earthquake generated peculiar surface waves that were observed in a wide area of Tohoku (Noguchi et al. 2016). We attempted to reconstruct the wavefield observed during this earthquake by applying seismic gradiometry to the Hi-net records.

Because Hi-net sensors have a natural frequency of $1 \mathrm{~Hz}$ and a relatively smaller sensitivity to lower frequency components, the sensor sensitivity at the stations is corrected by applying a time-domain digital deconvolution filter (Maeda et al. 2011). With this correction, it has been confirmed that the long-period component is well recovered for earthquakes with the magnitude of the 2005 event. We used the same period band (25-50 s) that we used for the synthetic tests. Spatial derivatives of the displacement records with respect to horizontal directions were estimated in the same manner as for the analyses of the synthetic traces. In the record section in this period band (Fig. 7), the arrivals of long-period $P$-waves and dispersive surface (Rayleigh) waves are clearly recognized. The Hi-net stations are equipped with velocity-type sensors, and the ground displacement traces were obtained by numerical integration over time. Then, divergence and rotation and the gradiometry parameters (Eq. 12) were estimated to obtain the slowness vector (Eq. 9). We used a 

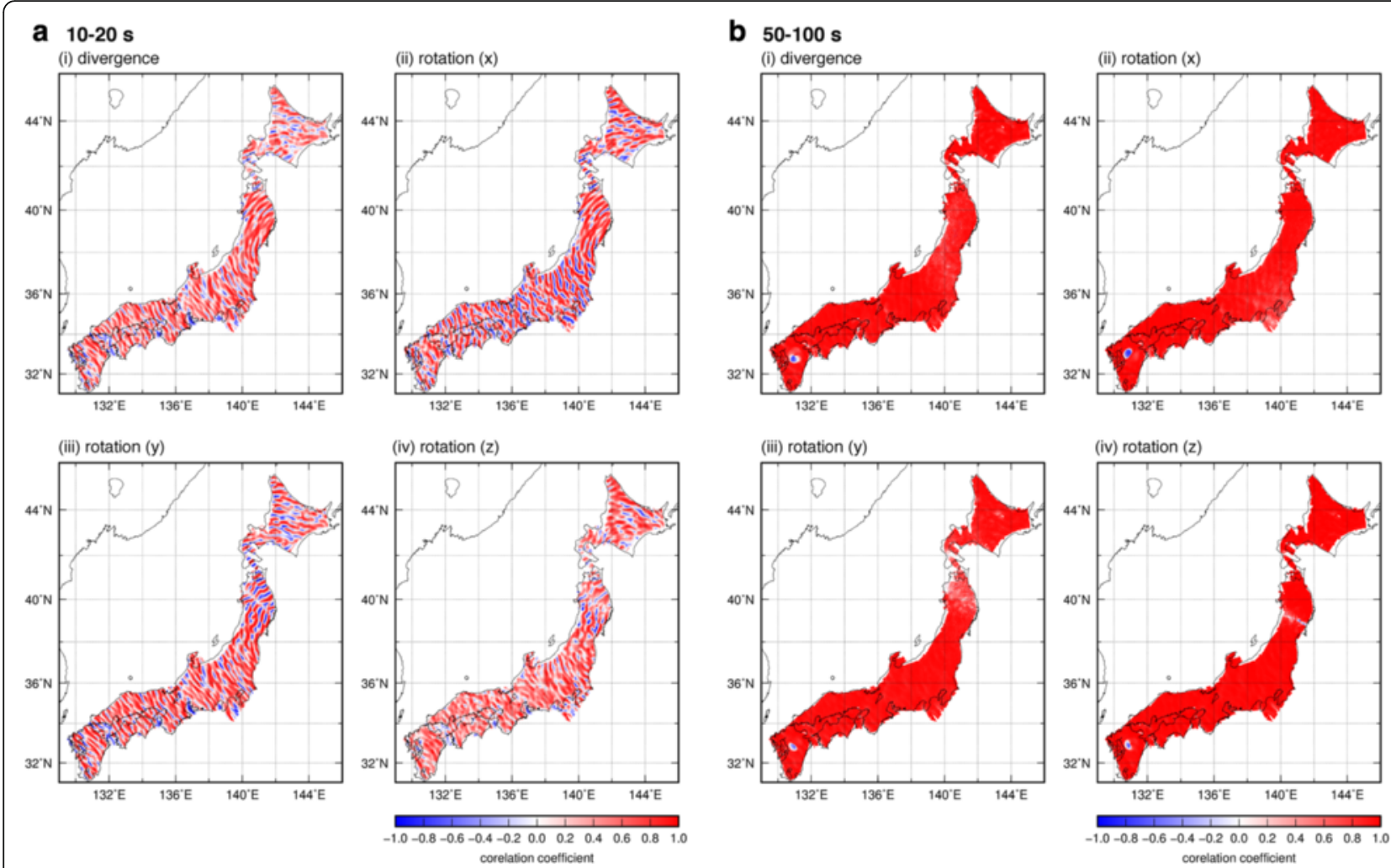

Fig. 6 Same as Fig. 4 but for the (a) 10-20 s and (b) 50-100 s period bands

grid spacing of $0.02^{\circ}$ for the gradiometry, which is finer than the spacing used for the synthetic test.

To estimate the gradiometry parameters, we used time windows with a duration of $75 \mathrm{~s}$, which is 1.5 times the maximum period of the analyzed period band of 25-50 s. This time window was shifted at intervals of $1 \mathrm{~s}$ to estimate the temporal variation of the slowness vector. Because we occasionally found that the estimation of the gradiometry parameters became unstable because the denominator of Eq. (12) approached zero, we set an empirical threshold for the denominator value as follows:

$$
\frac{\left(\boldsymbol{u}_{t} \cdot \boldsymbol{u}_{t}\right)\left(\boldsymbol{v}_{t} \cdot \boldsymbol{v}_{t}\right)-\left(\boldsymbol{u}_{t} \cdot \boldsymbol{v}_{t}\right)^{2}}{\left|u_{\max }\right|^{2}\left|\nu_{\max }\right|^{2}}>\epsilon
$$

where $\left|u_{\max }\right|$ and $\left|v_{\max }\right|$ are the absolute values of the maximum amplitude of ground displacement and ground velocity traces in the whole time series, and $\sim 10^{-6}$ is an empirically determined tolerance value. We estimated the gradiometry parameters when condition (13) was satisfied. This condition tended to not be met when the waves were not coherent at all, in particular, in the noise before the arrival of the first seismic wave. This result is consistent with the wavefield characterization in Eq. (8), for which it was assumed that a single wave packet dominated in the time window.

In real-world applications, it is also essential to avoid data from problematic stations. In particular, long-period seismograms recorded by short-period seismometers with correction of their sensor responses are sometimes very noisy, even if the high-frequency components of the raw seismograms seem to be normal. To deal with this problem in an automated way, we introduced an iterative method to exclude outliers based on the root-meansquared (RMS) amplitude of the noise level measured before the first arrival of the $P$-wave. First, the average and the standard deviations of this RMS amplitude were calculated, and the station having the largest standard deviation was excluded. In this procedure, only one station was excluded because a significant outlier may impact estimation of the average. Then, the average and standard deviations were calculated again using the dataset excluding the outlier. This procedure was repeated until all data were within three standard deviations of the average. This procedure is very similar in concept to the Smirnov-Grubbs statistical test (Grubbs 1969), but we used an empirical threshold rather than the test statistic, because the RMS amplitude may not follow a normal distribution.

Time-lapse snapshots of the reconstructed seismic wavefield and associated horizontal slowness vectors (Fig. 8) and 

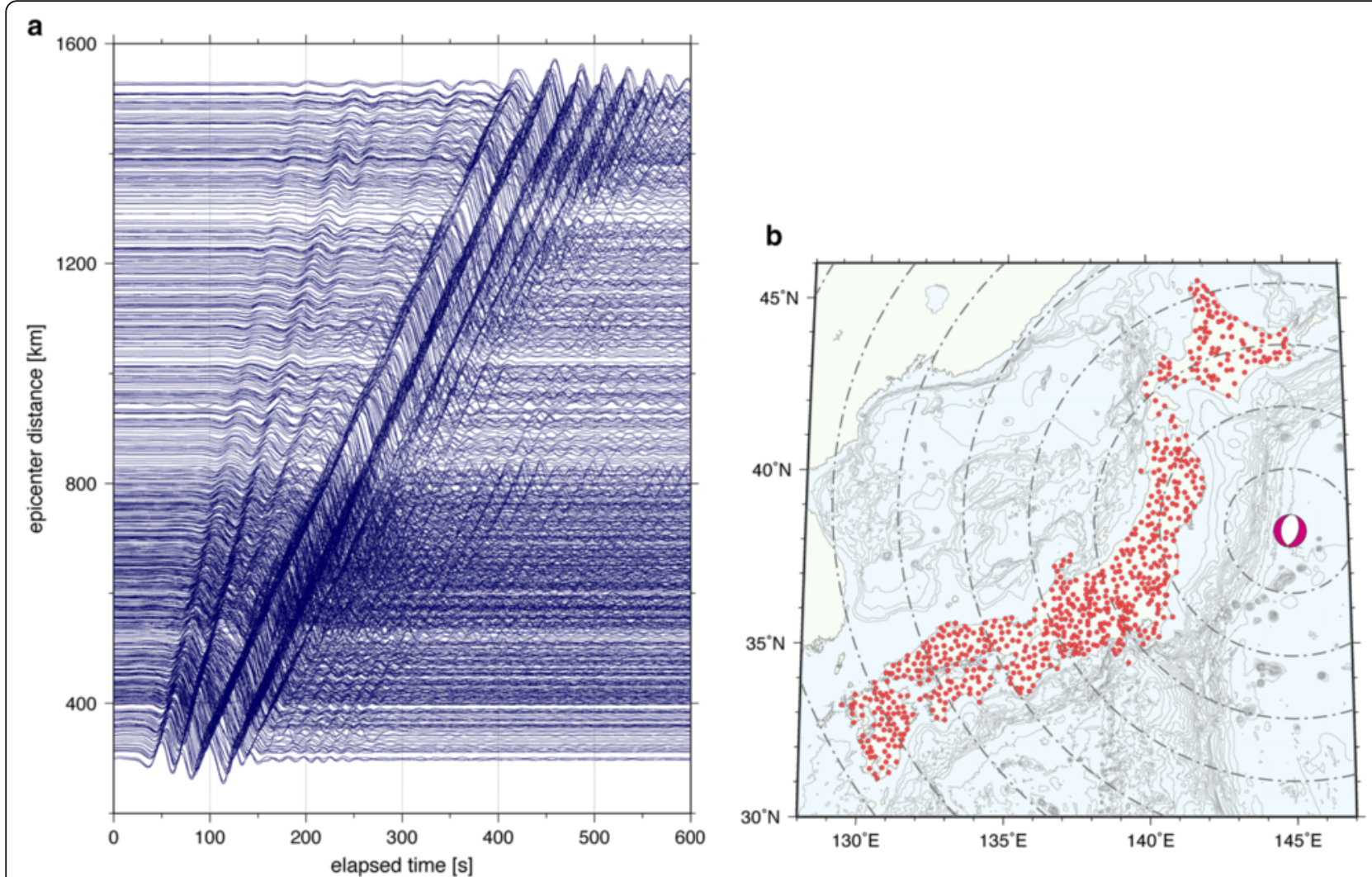

Fig. 7 a Vertical-component displacement traces at all Hi-net stations used in the analysis for the 2005 Off-Tohoku outer-rise earthquake at the 25-50 s period band. Amplitudes are normalized by the maximum value along each trace. $\mathbf{b}$ Station map with the earthquake mechanism from the Global CMT catalog. Light gray contours show bathymetry (contour interval, $500 \mathrm{~m}$ ). The gray dash-dot arcs indicate iso-distances at $200 \mathrm{~km}$ intervals from the epicenter

a time-sequential movie (Additional file 3: Movie S3) show that as the wave propagates, the systematic wave propagation direction is obtained as a spatially varying horizontal slowness vector. In the first time frame $(50 \mathrm{~s}$ of elapsed time; Fig. 8a), a continuous $P$-wave was clearly observed at individual stations (mark A1), and a smooth, continuous wavefield was reconstructed by seismic gradiometry (mark A2). In addition, propagation direction vectors with horizontal slowness amplitudes of $\sim 0.1 \mathrm{~s} / \mathrm{km}$, which clearly correspond to the reciprocal of the apparent wavespeed of $P$-waves, were widely distributed in NE Japan (mark A3). We obtained some peculiar behaviors characterized by unrealistically large slowness vectors with an inconsistent direction in the Kanto district (mark B1). We attributed these behaviors to two stations with inconsistent observations (mark B2). Notice that the propagation direction was random in SE Japan, where at $20 \mathrm{~s}$ the first $P$-wave had not yet arrived (mark C). We note that the propagation directions are coherently estimated in the middle part of Honshu Island, although the amplitude of the vertical component displacement appears to be very small. The small amplitude is partly due to the effect of finite time window of $75 \mathrm{~s}$.
At the elapsed time of $t=130 \mathrm{~s}$ (Fig. 8b), surface waves appeared near the epicenter in northeastern Japan. They were clearly recognized as a wave packet with wavelengths different from those of the body waves (marks D1 and E1). A corresponding difference in slowness was also seen, reflecting the difference between the apparent speed of the body waves and the phase speed of the surface waves (marks D2 and E2, respectively). At a late elapsed time $(t=230 \mathrm{~s}$; Fig. 8c), the dispersion of the Rayleigh waves was clearly recognized by a gradual change of wavelength (mark F; longer in the SW to shorter in the NE) in the reconstructed wavefield. It is noteworthy that the major arrival directions of body waves in western Japan and surface waves in central Japan were not perfectly homogeneous but fluctuated in space, probably reflecting heterogeneity beneath Japan.

In the divergence and rotation wavefields (Fig. 9), the direct $P$-wave was separated into divergence components (marks A1 and A2); Rayleigh and Love waves were also separated, having slightly different slownesses, as also occurred in the synthetic test. We also recognized a clear phase offset (mark B in Fig. 9b) of Love waves that appeared in the vertical component of the rotation vector at $t=120 \mathrm{~s}$. 


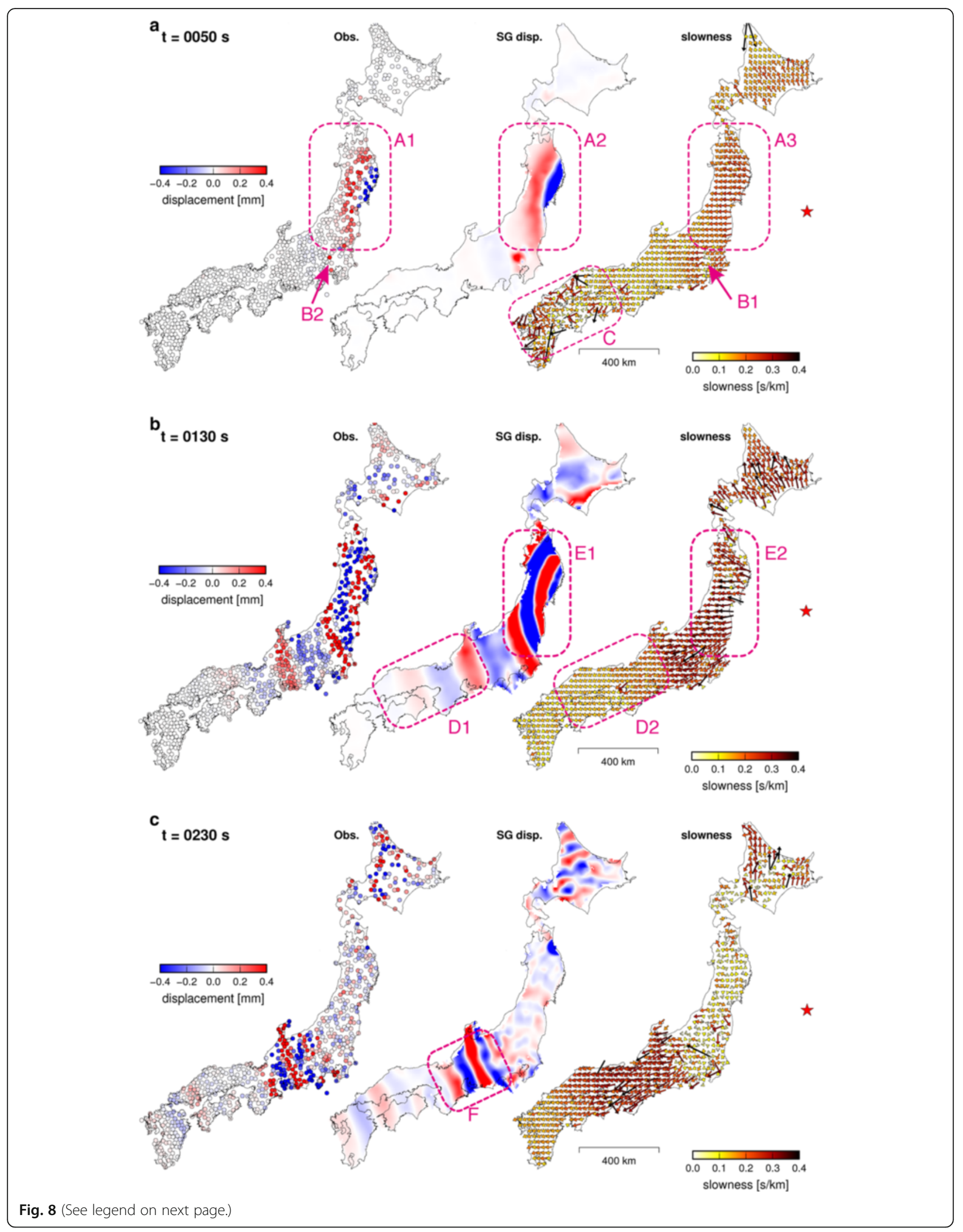


(See figure on previous page.)

Fig. 8 Wavefield and propagation direction analyzed by seismic gradiometry at elapsed times of $t=20 \mathrm{~s}(\mathbf{a}), t=130 \mathrm{~s}(\mathbf{b})$, and $t=230 \mathrm{~s}$ (c). Each panel shows the observed vertical-component displacement amplitude (color scale) at stations in the 25-50 s period band (left), the continuous displacement wavefield reconstructed by seismic gradiometry (center), and propagation direction and its slowness estimated from the gradiometry parameters (right). The absolute value of slowness is shown by the color scale. Arrow length is proportional to the absolute value of the slowness. The star in the right panel shows the epicenter location. An MP4-formatted movie of the time-dependent wavefield is included in Additional file 3: Movie S3

This offset is most likely an effect of the source radiation pattern. At a very late elapsed time $(t=300 \mathrm{~s})$, we observed non-great-circle path propagation in both divergence (mark C) and the vertical component of the rotation (mark D). Interestingly, the propagation directions of these wave packets differed between the divergence and rotation wavefields, suggesting either an individual scattering effect or a multi-path effect of Rayleigh and Love waves. We expect this divergence-rotation decomposition technique aided by seismic gradiometry to be helpful for investigating the constituents and origin of waves attributable to inhomogeneous structure.

We found that the divergence and rotation were more sensitive to problematic station data. At the locations marked E1-E4, the seismogram was always oscillatory irrespective of the arrival of seismic waves (see Additional file 4: Movie S4). The amplitudes of the displacements and the derived wavefield (Fig. 8 and Additional file 3: Movie S3) did not show peculiar behaviors at these locations. Although this oscillatory feature is most probably due to problematic stations, it was not possible to automate their detection in the amplitudes observed before $P$-wave arrival.

\section{Discussion}

Seismic gradiometry enables us to characterize the propagation direction by assuming that the wavefield consists of a single wave packet (Langston 2007b). Given this assumption, the method can successfully reproduce the major propagation direction and the slowness of direct waves and the early part of the surface wave coda. The estimated propagation direction of later coda was nearly random; thus, a more detailed investigation may be necessary to determine whether the assumption of a single wave packet is satisfied at later elapsed times. If the wavefield then consists of omni-directional arrivals, suggesting a diffuse state (Weaver 1982), then wavefield characterization by seismic gradiometry would not be applicable, at least in its current form. Characterization of multiple incoming waves by this method should be addressed by future studies.

On the other hand, estimation of divergence and rotation by seismic gradiometry, newly proposed in this study, is not restricted by assumptions about wave behavior. Their estimation is always valid as long as the wavefield is continuous in space and its derivatives are correctly estimated. We showed that the spatio-temporal pattern of divergence and rotation, in particular the vertical component of the rotation vector, are extremely useful for inferring the wave constituents. Comparison of the reconstructed ground displacement or ground velocity wavefield with these divergence and rotation wavefields should be helpful for investigating the constituents of wave packets originated from inhomogeneous structures (e.g., Obara and Matsumura 2010; Domínguez et al. 2011; Noguchi et al. 2013, 2016; Maeda et al. 2014).

In the real-world application, we found that the recovery of a continuous, coherent wavefield was better achieved for the vertical component of the rotation vector than for the divergence (Fig. 9). This difference may be because, for divergence, the estimation of the derivative with respect to depth is indirect, whereas for the vertical component of the rotation vector, it is estimated directly from the derivatives in the horizontal directions. Recall that the derivative with respect to depth is estimated under the assumption of a traction-free boundary condition, which is only approximately achieved at the Hi-net stations installed at the bottom of boreholes (Obara et al. 2005); as a result, stable estimation may be distorted. If that is the case, this boundary condition becomes more valid for longer wavelengths in long-period bands. This effect was apparently confirmed in the synthetic test (Fig. 6). The recovery of divergence was better in the long-period band of 50-100 s than in the 25-50 s period band (Fig. 5).

This study focused on reconstructing the wavefield as a new method of analyzing seismic wavefields. If we concentrate on the arrival of direct waves, wavefield characterization by using gradiometry parameters leads directly to the estimation of phase speed and the azimuthal variation of surface waves from the great circle path (Liang and Langston 2009), which eventually leads to the estimation of the three-dimensional inhomogeneous structure. Because the method provides a stable estimate of phase speed even from records of a single event, by compiling estimations by seismic gradiometry for many earthquakes having various back azimuth directions, it may be possible to analyze azimuthal anisotropy. Also, the use of the radiation pattern and geometrical spreading terms, which can be obtained from the gradiometry parameters, should be investigated in the future. 


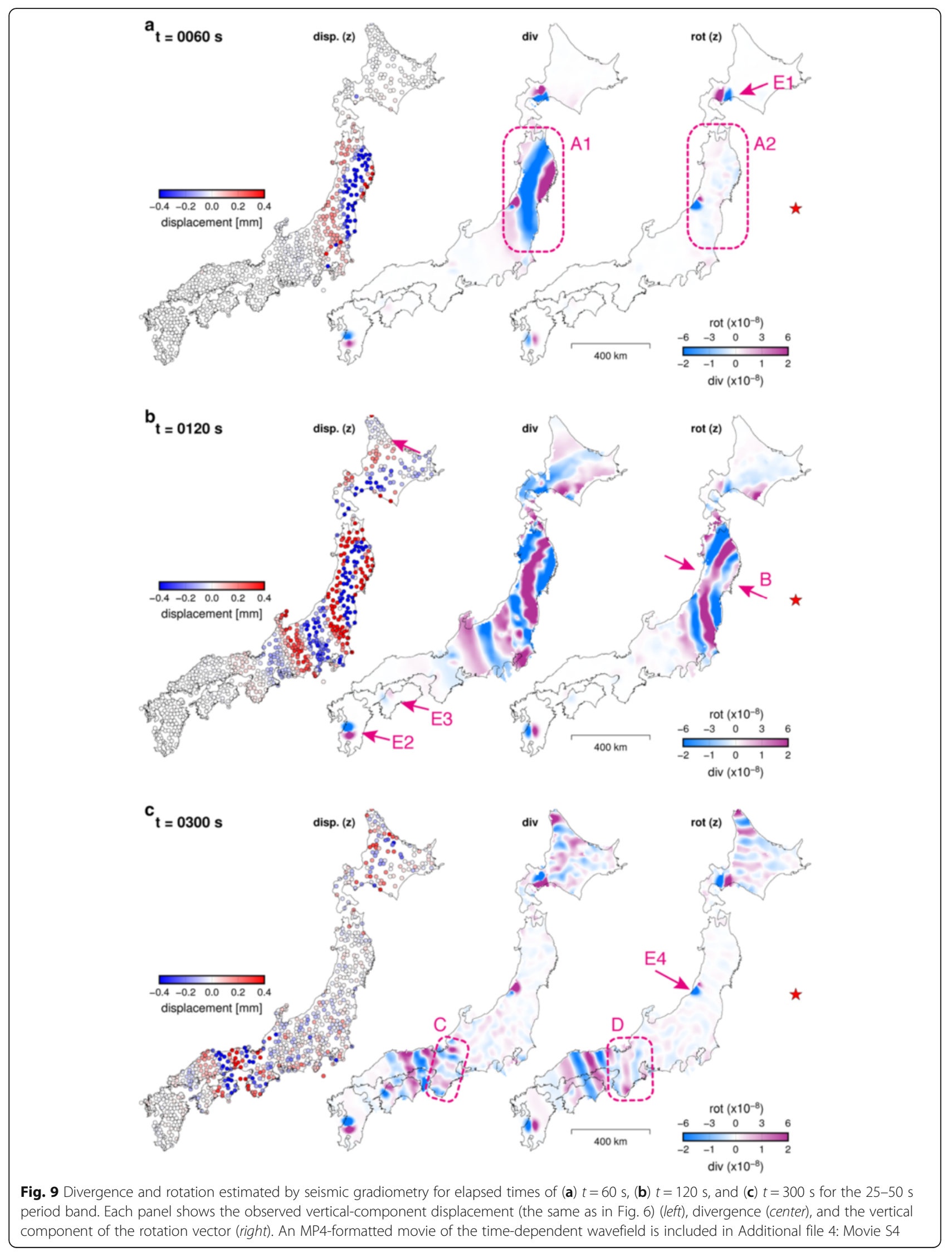


The estimation of the spatial derivative itself has room for further improvement. The first possible improvement is correction of site amplification. In high-frequency bands, the amplitudes observed at stations are significantly affected by local site amplification (Takemoto et al. 2012). Even at very low frequencies, where the surface wave mode dominates, the amplitude of the eigenfunction of surface waves is affected by local inhomogeneous structures near stations (Aki and Richards 2002). Although random fluctuation of the amplitude at stations can be minimized by least squares estimation of the spatial gradient, explicit correction of pre-estimated site amplification factors should allow more precise estimation of amplitude.

Because seismic gradiometry estimates slowness and other wave features from spatial gradients, its application might not be restricted to seismic waves in long-period bands. In high-frequency seismograms with $f>1 \mathrm{~Hz}$, the phase of the seismogram is usually very complicated owing to complicated wave scattering caused by smallscale inhomogeneity (Sato et al. 2012). Thus, applications of array methods to high-frequency seismograms are limited to records from local arrays of small spatial scale (e.g., Taira et al. 2007). However, seismogram envelopes are known to have similar shapes at nearby stations, and these characteristics have long been used for locating non-volcanic deep low-frequency tremors by the envelope correlation method (e.g., Obara 2002; Maeda and Obara 2009). We may therefore be able to apply the seismic gradiometry technique to seismogram envelope amplitudes in order to reconstruct the envelope wavefield and to estimate its propagation direction (see Appendix). The application of seismic gradiometry to seismogram envelope may be useful for investigating seismic wave energy flow in high-frequency bands.

The spatial modeling of waves could also be improved. We adopted a simple first-order Taylor series in this study. Recently, Mizusako et al. (2014) showed that wave amplitude and its derivatives, including higher order derivatives, could be well-estimated by applying the Least Absolute Shrinkage and Selection Operator (LASSO) to the station layout of the Metropolitan Seismic Observation network (MeSO-net) (Kasahara et al. 2009). They showed that the continuous wavefield and its derivatives were quite well-estimated by their method, even though the MeSO-net station layout is much more irregular than the Hi-net layout. Application of such cuttingedge mathematical technology will enable more accurate characterization of seismic waves, and, hence, the inhomogeneous structure beneath Japan.

\section{Conclusions}

In this paper, we attempted to visualize and characterize seismic wave traces at individual stations by using seismic gradiometry. The use of seismic gradiometry has three major benefits: (i) it enables us to treat seismic waves as a continuous 2D seismic wavefield rather than as a set of individual traces; (ii) the estimation of spatial derivatives enables us to separate divergence and rotation vector elastic oscillation; and (iii) it is useful for accurate estimation of slowness vectors as spatially varying field data.

Although wavefield visualization from seismic sensors has been done by applying spatial smoothing (Sheldrake et al. 2002; Maeda et al. 2014) or by using a set of individual traces (Trabant et al. 2012), seismic gradiometry makes it possible to estimate spatial gradients in horizontal directions as well, and these horizontal gradients are extremely useful for characterizing wave propagation. Also, the estimation process is linear and therefore can be done very quickly; thus, it is suitable for (semi) real-time seismic wavefield monitoring.

The results of our numerical experiments using finite difference simulations with a realistic $3 \mathrm{D}$ inhomogeneous medium model, together with the application of the method to real-world data, show that we can reconstruct a continuous and coherent wavefield with a period longer than $25 \mathrm{~s}$ by applying seismic gradiometry to $\mathrm{Hi}$-net records. Because it was previously confirmed that $\mathrm{Hi}$-net stations are capable of recording such long-period data for far-field earthquakes of magnitude larger than 7 (Maeda et al. 2011), this method should be applicable to any such large earthquake if the records are not clipped by strong ground motion.

\section{Appendix}

A trial application of seismic gradiometry to high-frequency seismogram envelopes

In this appendix, we attempt to apply the seismic gradiometry method to a seismogram envelope in highfrequency bands to extract the propagation features. In its original form, the gradiometry equation requires ground velocity and ground displacement and its spatial derivatives in horizontal directions to be known (see Eq. 9). We decided to use the seismogram envelope as the ground velocity term in this equation; the seismogram envelope integrated with respect to time was used as the displacement term in (Eq. 9) (hereinafter, the envelope displacement). The spatial gradient of the envelope displacement amplitude rather than that of the envelope amplitude itself is estimated. After obtaining and estimating these values of the envelope and the envelope displacement and its spatial derivatives by the least squares method (Eq. 4), we estimated the gradiometry parameters in the same manner as for the waveforms in the application of seismic gradiometry to the long-period band. 


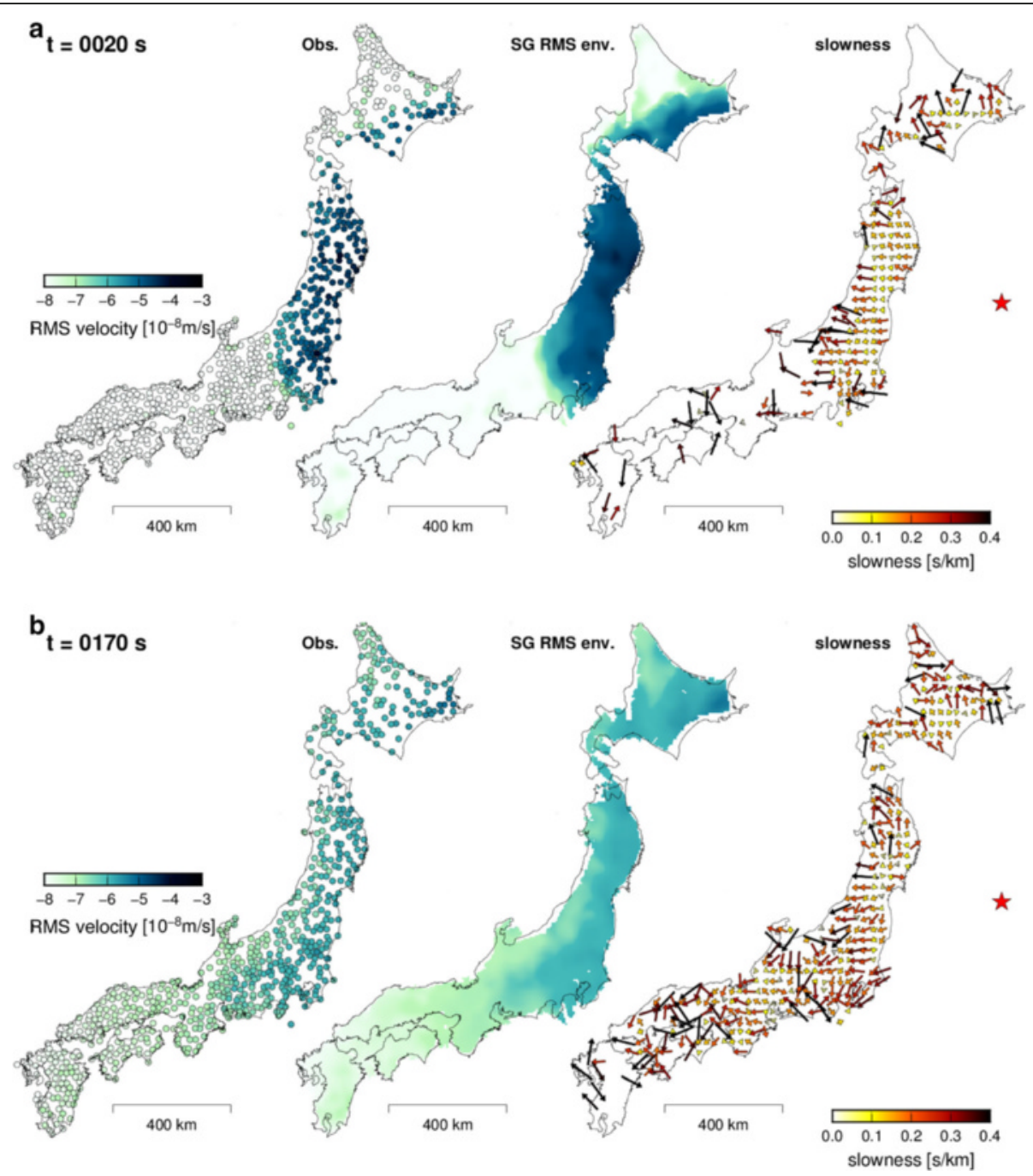

Fig. 10 Same as Fig. 6, but for the high-frequency $(2-8 \mathrm{~Hz})$ seismogram envelope at two elapsed times: (a) $t=20 \mathrm{~s}$ and (b) $t=170 \mathrm{~s}$. In each frame, the left panel shows the RMS envelope amplitude, the center panel shows the envelope field reconstructed by the seismic gradiometry, and the right panel shows the slowness vectors estimated from the envelope. An MP4-formatted movie of the time-dependent wavefield is included in Additional file 5: Movie S5

We tested this approach against real observations of a high-frequency seismogram recorded during the same earthquake in the $2-8 \mathrm{~Hz}$ frequency range (Fig. 10 and Additional file 5: Movie S5). Because the estimation was relatively unstable compared with the long-period band estimation, we used a time window as long as $75 \mathrm{~s}$. The adoption of a longer time window stabilized the analysis, although the estimation was still occasionally unstable owing to violations of the condition of Eq. (13). In particular, before the arrival of direct waves, systematic gradiometry parameter estimation was almost impossible. As shown in Fig. 10a, the slowness vectors at grid points were successfully estimated for the arrival of $P$-waves, and they indeed show that reasonable propagation directions, outgoing from the source, can be estimated from envelope records of incoherent high-frequency records.
After the arrival of the direct waves, the distribution of the slowness vectors became complicated but not random (right panel of Fig. 10b). The locally varying slowness directions are accompanied by somewhat large $(\sim 0.4 \mathrm{~s} / \mathrm{km}$ or more) slowness values, which is not realistic for the propagation of body waves. As shown by the reconstructed envelope amplitude (Fig. 10b), the envelope amplitude is still largest near the epicenter. This result is expected from the characteristics of the multiply scattered wave energy (e.g., Sato et al. 2012), in which the energy remains dominantly in the region around the hypocenter. The intrinsic attenuation of the seismic energy has considerable spatial inhomogeneity (Yoshimoto et al. 2006). This spatial inhomogeneity leads to changes in the spatial gradient of the seismogram envelope, which might be detected as apparent energy flow in the seismic gradiometry. 


\section{Additional files}

Additional file 1: Movie S1 (MP4 $13802 \mathrm{~kb}$ )

Additional file 2: Movie S2 (MP4 $2476 \mathrm{~kb}$ )

Additional file 3: Movie S3 (MP4 $6558 \mathrm{~kb}$ )

Additional file 4: Movie S4 (MP4 $3168 \mathrm{~kb}$ )

Additional file 5: Movie S5 (MP4 $1564 \mathrm{~kb})$

\section{Abbreviations}

Hi-net: High-sensitivity seismograph network of Japan; JVVSM: Japan integrated velocity structure model; MeSO-net: Metropolitan Seismic Observation network; NIED: National Research Institute for Earth Science and Disaster Resilience, Japan

\section{Acknowledgements}

We thank the data center of NIED for providing high-quality seismic data from Hi-net. The hypocenter location was obtained from the Japan Meteorological Agency hypocenter catalog. The Japan Integrated Velocity Structure Model (Koketsu et al. 2012) with modifications was used as the structural model for the numerical simulation. We used the gridded bathymetry dataset JTOPO30v2 provided by the Marine Information Research Center, Japan Hydrographic Association. The numerical simulation in this study was performed with the ElC computer system at the Earthquake Information Center of the Earthquake Research Institute, the University of Tokyo. We express our gratitude to Nori Nakata and an anonymous reviewer for their insightful comments and suggestions. This work was partly supported by the Earthquake Research Institute Cooperative Research Program (2015-B-01).

\section{Funding}

TM was funded by the Japan Society for the Promotion of Science (JSPS), KAKENHI Grant number15 K16306.

\section{Authors' contributions}

$\mathrm{TM}, \mathrm{KN}$, and RT participated in the theoretical development, and TM carried out the data analysis and numerical simulation. KO conceived the study and contributed to the concepts and design of important intellectual components of the paper. All authors contributed to the drafting of the manuscript. All authors read and approved the final manuscript.

\section{Competing interests}

The authors declare that they have no competing interests.

\section{Author details}

${ }^{1}$ Earthquake Research Institute, The University of Tokyo, 1-1-1 Yayoi, Bunkyo-ku, Tokyo 113-0032, Japan. ${ }^{2}$ Research Center for Prediction of Earthquakes and Volcanic Eruptions, Graduate School of Science, Tohoku University, Sendai 980-8578, Japan.

Received: 22 May 2016 Accepted: 20 September 2016

Published online: 10 October 2016

\section{References}

Aki K, Richards PG (2002) Quantitative seismology, 2nd edn. University Science Books, California

Domínguez LA, Sánchez-Sesma FJ, Davis PM (2011) Scattering of teleseismic body waves by the lateral crustal heterogeneity at the Pacific trench of Mexico. Bull Seism Soc Am 101(3):1281-1290. doi:10.1785/0120100181

Grubbs FE (1969) Procedures for detecting outlying observations in samples. Technometrics 1969:1-21. doi:10.1080/00401706.1969.10490657

Igel H, Schreiber U, Flaws A, Schuberth B, Velikoseltsev A, Cochard A (2005) Rotational motions induced by the M8.1 Tokachi-oki earthquake, September 25, 2003. Geophys Res Lett 32(8):L08309. doi:10.1029/2004GL022336

Kasahara K, Sakai S, Morita Y, Hirata N, Tsuruoka H, Nakagawa S, Nanjo KZ, Obara K (2009) Development of the metropolitan seismic observation network (MeSO-net) for detection of mega-thrust beneath Tokyo metropolitan area. Bull Earthq Res Inst Univ Tokyo 84:71-88 (in Japanese with English Abstract)

Koketsu K, Miyake H, Suzuki H (2012) Japan integrated velocity structure model version 1. Proc WCEE15 paper number 1773.

Kumagai H, Pulido N, Fukuyama E, Aoi S (2012) Strong localized asperity of the 2011 Tohoku-Oki earthquake, Japan. Earth Planets Space 64(7):649-654. doi:10.5047/eps.2012.01.004
Langston CA (2007a) Spatial gradient analysis for linear seismic arrays. Bull Seism Soc Am 97(1B):265-280. doi:10.1785/0120060138

Langston CA (2007b) Wave gradiometry in two dimensions. Bull Seism Soc Am 97(2):401-416. doi:10.1785/0120060138

Langston CA (2007c) Wave gradiometry in the time domain. Bull Seism Soc Am 97(3):926-933. doi:10.1785/0120060152

Langston CA, Liang C (2008) Gradiometry for polarized seismic waves. J Geophys Res 113(B8):B08305. doi:10.1029/2007JB005486

Liang C, Langston CA (2009) Wave gradiometry for USArray: Rayleigh waves. J Geophys Res 114(B2):B02308. doi:10.1029/2008JB005918

Liu Y, Holt WE (2015) Wave gradiometry and its link with the Helmholtz equation solutions applied to US Array in the eastern U.S. J Geophys Res 120:5717-5746. doi:10.1002/2015JB011982

Maeda T, Furumura T (2013) FDM Simulation of seismic waves, ocean acoustic waves, and tsunamis based on tsunami-coupled equations of motion. Pure Appl Geophys 170(1-2):109-127. doi:10.1007/s00024-011-0430-z

Maeda T, Obara K (2009) Spatiotemporal distribution of seismic energy radiation from low-frequency tremor in western Shikoku, Japan. J Geophys Res 114(B10):B00A09. doi:10.1029/2008JB006043

Maeda T, Obara K, Furumura T (2011) Interference of long-period seismic wavefield observed by the dense Hi-net array in Japan. J Geophys Res 116(B10):B10303. doi:10.1029/2011JB008464

Maeda T, Furumura T, Noguchi S, Takemura S, Sakai S, Shinohara M, Iwai K, Lee S-J (2013) Seismic- and tsunami-wave propagation of the 2011 off the Pacific coast of Tohoku earthquake as inferred from the tsunami-coupled finite-difference simulation. Bull Seism Soc Am 103(2B):1456-1472. doi:10.1785/0120120118

Maeda T, Furumura T, Obara K (2014) Scattering of teleseismic P-waves by the Japan Trench: a significant effect of reverberation in the seawater column. Earth Planet Sci Lett 397:101-110. doi:10.1016/j.epsl.2014.04.037

Mavroeidis GP, Papageorgiou AS (2003) A mathematical representation of near-fault ground motions. Bull Seism Soc Am 93(3):1099-1131. doi:10.1785/0120020100

Menke W (2012) Geophysical data analysis: discrete inverse theory, third Edition. Academic Press, Cambridge, Massachusetts

Mizusako S, Nagao H, Hirose K, Kano M, Hori M (2014) Imaging ground motions in the Tokyo metropolitan area based on MeSO-net using LASSO. Proceedings of the 34th Conference on Earthquake Engineering. (in Japanese with English abstract)

Neidell NS, Taner MT (1971) Semblance and other coherency measures for multichannel data. Geophysics 36(3):482-497. doi:10.1190/1.1440186

Noguchi S, Maeda T, Furumura T (2013) FDM simulation of an anomalous later phase from the Japan trench subduction zone earthquakes. Pure Appl Geophys 170(1-2):95-108. doi:10.1007/s00024-011-0412-1

Noguchi S, Maeda T, Furumura T (2016) Ocean-influenced Rayleigh waves from outer-rise earthquakes and their effects on durations of long-period ground motion. Geophys J Int 205(2):1099-1107. doi:10.1093/gji/ggw074

Obara K (2002) Nonvolcanic deep tremor associated with subduction in southwest Japan. Science 296(5573):1679-1681. doi:10.1126/science.1070378

Obara K, Matsumura M (2010) Reverse propagation of surface waves reflected from seamounts in the Northwestern Pacific. Bull Seism Soc Am 100(3):1342-1349. doi:10.1785/0120090291

Obara K, Kasahara K, Hori S, Okada Y (2005) A densely distributed high-sensitivity seismograph network in Japan: Hi-net by national research institute for earth science and disaster prevention. Rev Sci Inst 76(2):021301. doi:10.1063/1.1854197

Okada Y, Kasahara K, Hori S, Obara K, Sekiguchi S, Fujiwara H, Yamamoto A (2004) Recent progress of seismic observation networks in Japan-Hi-net, F-net, K-NET and KiK-net-. Earth Planets Space 56(8):xv-xxviii. doi:10.1186/ BF03353076

Sato H, Fehler MC, Maeda T (2012) Seismic wave propagation and scattering in the heterogeneous earth, 2nd edn. Springer, Berlin Heidelberg

Shapiro NM, Campillo M, Margerin L, Singh SK, Kostoglodov V, Pacheco J (2000) The energy partitioning and the diffusive character of the seismic coda. Bull Seism Soc Am 90(3):655-665. doi:10.1785/0119990021

Sheldrake KP, Marcinkovich C, Tanimoto T (2002) Regional wavefield reconstruction for teleseismic P-waves and surface waves. Geophys Res Lett 29(11):1544. doi:10.1029/2001GL013721

Spudich P, Steck LK, Hellweg M, Fletcher JB, Baker LM (1995) Transient stresses at Parkfield, California, produced by the M7.4 Landers earthquake of June 28, 1992: observations from the UPSAR dense seismograph array. J Geophys Res 100(B1):675-690. doi:10.1029/94JB02477

Stein S, Wysession M (2003) An introduction to seismology, earthquakes, and earth structure. Blackwell Publishing, Malden 
Taira T, Yomogida K, Kuwahara Y, Imanishi K, Ito H (2007) Imaging of crustal heterogeneous structures using a slowness-weighted back-projection with effects of scattering modes: 2. Application to the Nagamachi-Rifu fault, Japan, area. J Geophys Res 112(B6):B06312. doi:10.1029/2006JB004382

Takemoto T, Furumura T, Saito T, Maeda T, Noguchi S (2012) Spatial- and frequency-dependent properties of site amplification factors in Japan derived by the coda normalization method. Bull Seism Soc Am 102(4):1462-1476. doi:10.1785/0120110188

Trabant C, Hutko AR, Bahavar M, Karstens R, Ahern T, Aster R (2012) Data products at the IRIS DMC: stepping stones for research and other applications. Seism Res Lett 83(5):846-854. doi:10.1785/0220120032

Weaver RL (1982) On diffuse waves in solid media. J Acoust Soc Am 71(6):1608-1609. do:10.1121/1.387816

Yoshimoto K, Wegler U, Korn M (2006) A volcanic front as a boundary of seismicattenuation structures in northeastern Honshu, Japan. Bull Seism Soc Am 96(2):637-646. doi:10.1785/0120050085

\section{Submit your manuscript to a SpringerOpen ${ }^{\circ}$ journal and benefit from:}

- Convenient online submission

- Rigorous peer review

- Immediate publication on acceptance

- Open access: articles freely available online

- High visibility within the field

- Retaining the copyright to your article

Submit your next manuscript at $\gg$ springeropen.com 